\title{
Aligning and Comparing Data on Emotions Experienced during Learning with MetaTutor
}

\author{
Jason M. Harley, François Bouchet, and Roger Azevedo \\ McGill University, Laboratory for the Study of Metacognition and \\ Advanced Learning Technologies, Montreal, Canada \\ jason.harley@mail.mcgill.ca
}

\begin{abstract}
In this study we aligned and compared self-report and on-line emotions data on 67 college students' emotions at five different points in time over the course of their interactions with MetaTutor. Self-reported emotion data as well as facial expression data were converged and analyzed. Results across channels revealed that neutral and positively-valenced basic and learnercentered emotional states represented the majority of emotional states experienced with MetaTutor. The self-report results revealed a decline in the intensity of positively-valenced and neutral states across the learning session. The facial expression results revealed a substantial decrease in the number of learners' with neutral facial expressions from time one to time two, but a fairly stable pattern for the remainder of the session, with participants who experienced other basic emotional states, transitioning back to a state of neutral between self-reports. Agreement between channels was $75.6 \%$.
\end{abstract}

Keywords: Emotions, affect, intelligent tutoring systems, pedagogical agents.

\section{Emotions during Learning with ITSs}

Effective learning and students' experience of emotions are critically related [e.g., $1,2]$. For ITS research, this translates into a recognized need to design systems with embodied pedagogical agents (PAs) that use AI algorithms to detect, model, and adapt to changes in learners' emotional fluctuations, in order to promote adaptive emotional states that will facilitate learning [3-5]. Despite the recent surge in interdisciplinary research on emotions and affective computing [6], little is known about many important facets of learners' emotional experiences with ITSs, such as how learners' emotions fluctuate over time (e.g., over the course of a learning session) and how different components (behavioral, physiological, and experiential) of emotions align. Identifying patterns in learners' emotional experiences over time is critical to understanding how learners' feel as they progress temporally through the learning session. In particular, such finer-grained analyses provide valuable diagnostic information regarding events or time segments to focus system changes on, such as changes to the rules used to determine system dynamics or the creation of new PA-delivered emotional interventions. It is equally paramount to assess the convergence of different methods for measuring emotions in order to establish convergent 
validity between methodologies and to further our psychological theories of emotions regarding, for example, the loose or tight coupling of different emotional expression components [7]. Answering these questions will help ITS researchers design more effective emotionally adaptive ITSs with improved calibration between the emotionregulating prompts provided by PAs and learners' emotional states. Furthermore, this important user-diagnostic information will also help reduce the negative outcomes associated with mis-calibrations between participants' experienced emotional states and ITSs' understanding of them [3-5].

\subsection{Research Objectives}

There were three primary purposes of this study. (1) To examine learners' emotional responses across the MetaTutor learning session to determine which emotions were most prominently experienced and whether they changed as the learning session unfolded. (2) To examine whether significant differences in learners' emotional experiences existed between MetaTutor's two PAs scaffolding conditions: prompt and feedback (PF) and control (C). (3) To examine whether there was convergent evidence of learners' emotional experiences between the two emotion measurement methods we used: automatic facial expression analysis (FaceReader 5.0 [8]), and an in-session, concurrent, emotional state self-report measure (Emotions-Value questionnaire).

\section{Methods}

\subsection{Participants}

67 undergraduate students from a large, public university in North America participated in this study. Participants (82.8\% female, $72.4 \%$ Caucasian) were randomly assigned to either the $\mathrm{C}$ or $\mathrm{PF}$ condition.

\subsection{MetaTutor and Apparatus}

MetaTutor [9] is a multi-agent ITS and hypermedia learning environment which consists of 38 pages of text and static diagrams organized by a table of contents displayed in the left pane of the environment. The version of MetaTutor used in this experiment is comprised of material on the human circulatory system, which it is designed to teach participants about during their interactions with four embedded, pedagogical agents (PAs). The four PAs' instructional scaffolding varied depending on the experimental condition learners were assigned to (aside from PA scaffolding, the $\mathrm{C}$ and PF conditions were identical). In the PF condition, learners were prompted by the PAs to use specific self-regulatory processes (e.g., to metacognitively monitor their emerging understanding of the topic or deploy a specific cognitive learning strategy such as re-reading or coordinating informational sources), and were given feedback about their use of those processes. In the $\mathrm{C}$ condition, participants did not receive prompts or feedback. 
A Logitech Orbit AF webcam was used to record the participants' faces during their interaction with MetaTutor. In accordance with FaceReader's guidelines, the camera was mounted above the monitor of the computer participants were using, in order to capture their faces, but not obstruct the screen. Videos were recorded as WMV files with a resolution of $1600 \times 1200$, and 12.1 frames per second on average.

\subsection{Measures and Materials}

FaceReader 5.0. FaceReader [8] analyzes participants' facial expressions and provides a classification of their emotional states using an Active Appearance Model which models participants' facial expressions, and an artificial neural network with seven discrete outputs, corresponding to Ekman and Friesen's six basic emotions [10] in addition to neutral, that classifies participants' constellations of facial expressions. FaceReader has been validated through comparison with human coders [11]. Videos recorded during the two sessions of the experiment (with an average length of 40 and 100 minutes respectively) were imported and used to calibrate FaceReader with General or Asian face models. Videos of the second session (when the learning occurred) were then analyzed with the "smoothen classification" parameter enabled.

Emotions-Value Questionnaire (EV). During the learning session, participants were asked on five occasions (see section 2.4) by a PA to complete the EV questionnaire, for which each participant responded to 20 items: 19 items on emotions and 1 item on task value which was not considered in this analysis. These items were on a 5-point Likert scale ranging from "Strongly Disagree" to "Strongly Agree." One example item is: "right now I feel engaged." The 19 emotions that are measured using the EV represent an exhaustive list of discrete basic and learner-centered emotions that appear in the research and theories of a variety of emotion researchers [e.g., 2, 12]. Definitions, based on these researchers' work and operationalizations of these emotions, were used to create a digital, definition hand out that was provided in a side panel to participants every time they filled out an electronic version of the EV embedded in MetaTutor. The instructions and wording of the questions were based on a subscale of Pekrun et al. [13] academic emotions questionnaire (AEQ) which assesses participants' concurrent, 'right now' state-emotions as opposed to emotions generated from prospective or retrospective focal points. The majority of the 19 emotions can be conceptualized into different quadrants along the axis of valence (positive/negative) and activation (activating/deactivating) [2, 13].

\subsection{Experimental Procedure}

During Day One of the experiment, which took approximately 30 minutes, participants read and signed the informed consent form, took a pretest on the human circulatory system, completed a demographics questionnaire, and several self-report measures (e.g., AEQ trait emotions) on a computer with their face being video recorded. For Day Two, we collected video, audio, eye-tracking, and physiological data on each participant while they used MetaTutor for about $90 \mathrm{~min}$ to learn about 
the human circulatory system. At the beginning of the learning session participants set up two sub goals for learning about the human circulatory system and proceeded to interact with MetaTutor and its learning content for one hour; half-way through, they were asked to complete the concurrent state AEQ and then invited to take a fiveminute break. At the end of their learning session, learners filled out the post-test measure and a series of self-report measures, including the retrospective state AEQ. Days One and Two occurred at least one hour apart from each other and no more than four days apart. The first time participants filled out the EV was at the beginning of the learning session after they had successfully set two sub goals. The following occasions occurred regularly every 14 minutes during the on hour learning session, with the fifth EV being administered just before learners' took the post-test. Participants had as much time as necessary to fill out the EV on each occasion.

\subsection{Data Analysis}

FaceReader 5.0. FaceReader provides a score between 0 and 1, for each frame of each participant's video for each of Ekman's six basic emotions, in addition to neutral. FaceReader also provides information about the dominant emotional state (computed with a proprietary algorithm using the scores of the seven emotional states in the previous frames) and timestamp information regarding the on and offset of the hierarchical rankings of these states. In these analyses, we aligned FaceReader's dominant state with the EV by extracting log information corresponding to the 10 seconds of video footage of participants right before they were asked to fill in each of the EVs. We selected the primary dominant state defined as the state reported as dominant during the majority of the 10 seconds. In $80.7 \%$ of the cases, no other unique emotion was dominant for more than $3 \mathrm{~s}$, which makes it unnecessary to consider the possibility of a secondary co-occurring emotion [14]. Moreover, in $92.9 \%$ of the remaining situations, neutral was either the primary or secondary dominant emotion.

67 participants were analyzed, but nine of them were excluded from our sample because their dominant state in the 10s for at least three of the five EVs were identified as "Unknown" by FaceReader (this situation generally occurs when the participant's face is not sufficiently oriented towards the webcam, e.g. when they look down to type on the keyboard).

In order to evaluate the agreement between the self-reported emotions in the $5 \mathrm{EVs}$ and the dominant emotion identified by FaceReader during the 10s before, we started by defining a mapping between the 13 non-basic emotions from the EV onto the 6 basic emotions in addition to neutral that are used by FaceReader to classify participants' emotions. Using work from Pekrun et al. [2, 13] on the AEQ, (1) all positively valenced activating emotions (enjoyment, hope, pride, curiosity and eureka) were associated with happy; among the negatively valenced activating emotions, (2) frustration was grouped with anger, (3) anxiety with fear and (4) contempt with disgust, and (5) all negatively valenced deactivating emotions (hopelessness and boredom) were associated with sadness, while the (6 and 7) non-valenced emotions (neutral and surprise) were kept as two distinct categories. Two additional emotions (confusion and shame) used in the EV could not be associated to any basic emotions and were therefore discarded for this analysis. 
Given these seven groups of emotions, we defined that there was an agreement between FaceReader's dominant emotion and the EV if and only if one of the emotions associated to FaceReader's dominant emotion was rated with a score of 3 or more (out of 5) in the EV (e.g., if the dominant emotion according to FaceReader is anger, either anger or frustration need to have a score of 3 or more in the EV). The 20 (out of 290) occurrences of "Unknown" were excluded from this analysis.

EV. Several scores on different emotions on the EV measure were identified as univariate outliers with standardized scores exceeding $z=+/-3.29$ and were therefore replaced with the next most outlying values for each variable [15]. Several variables were identified as being skewed with values exceeding $z=+/-3.20$. Only emotion variables that were skewed across all five EVs were transformed, including fear, shame, hopelessness, disgust, sadness, and eureka. Square root, logarithmic, and inverse transformations were performed, but did not normalize the distributions for all variables (only hopelessness and eureka). Two to three of the five EV variables for anger, contempt, surprise, and confusion were skewed, but were not transformed in order to maintain consistency across the measures of each emotion.

\section{$3 \quad$ Results}

\subsection{Which Emotions Were Most Prominent in Learners' Experience with MetaTutor and Did They Change during a One-Hour Learning Session?}

Emotion-Value Questionnaire. We ran 19 repeated measure ANOVAs on the level of each self-reported emotion between the two conditions and across the five EVs. Table 1 provides the means and standard deviations (SDs) of each of the 19 emotions for each of the five EVs. Neutral $(M=3.36 ; S D=0.64)$, curiosity $(M=2.93 ; S D=0.71)$, and hope $(M=2.89 ; S D=0.54)$ had the highest mean levels when averaging all the EVs together. The inferential results of the repeated measure ANOVAs, summarized in Table 2, illustrate that the administration of the EV exerted a significant main effect on learners' experience of happiness, enjoyment, hope, pride, anger, frustration, surprise, confusion, curiosity, and neutral. In the interest of space, only significant results are reported in Table 2. Pairwise difference tests, conducted using a Bonferoni correction, revealed which EVs learners' emotions significantly differed between.

FaceReader. Table 3 provides a summary of the results obtained from FaceReader in which the frequencies and proportions of participants' dominant emotions are reported for each EV. Figure 1 illustrates the proportions from Table 3 using different gradients of circle sizes. Line gradients represent the number of participants who transition from one basic emotion state to another. For example, in the $10 \mathrm{sec}$. before participants reported their emotions on EV1, more than $50 \%$ of them (which we know to be $77.6 \%$ from Table 3 ) had a neutral facial expression. The thin solid blue lines show 
Table 1. Summary of means and standard deviations on emotions using the Evs

\begin{tabular}{|c|c|c|c|c|c|c|c|c|c|c|c|c|}
\hline \multirow[t]{2}{*}{ Emotion } & \multicolumn{2}{|r|}{1} & \multicolumn{2}{|r|}{2} & \multicolumn{2}{|c|}{3} & \multicolumn{2}{|c|}{4} & \multicolumn{2}{|c|}{5} & \multicolumn{2}{|c|}{ Avg. } \\
\hline & $\bar{M}$ & $S D$ & $M$ & $S D$ & $M$ & $S D$ & $M$ & $S D$ & $M$ & $S D$ & $M$ & $S D$ \\
\hline Happy & 3.03 & 0.67 & 2.90 & 1.00 & 2.62 & 0.93 & 2.59 & 0.97 & 2.59 & 1.12 & 2.74 & 0.48 \\
\hline Enjoy. & 3.07 & 0.95 & 2.91 & 1.14 & 2.66 & 1.00 & 2.57 & 1.06 & 2.50 & 1.05 & 2.74 & 0.52 \\
\hline Hope & 3.38 & 0.88 & 3.07 & 1.06 & 2.74 & 0.94 & 2.69 & 1.05 & 2.57 & 0.06 & 2.89 & 0.54 \\
\hline Pride & 2.74 & 0.81 & 2.67 & 0.98 & 2.38 & 0.95 & 2.48 & 1.05 & 2.40 & 0.97 & 2.53 & 0.51 \\
\hline Anger & 1.41 & 0.72 & 1.67 & 0.91 & 1.74 & 1.02 & 1.95 & 1.08 & 1.62 & 0.95 & 1.68 & 0.41 \\
\hline Frust. & 1.99 & 1.25 & 2.16 & 1.27 & 2.41 & 1.41 & 2.60 & 1.34 & 2.28 & 1.36 & 2.29 & 0.63 \\
\hline Anx. & 2.34 & 1.09 & 2.31 & 1.26 & 2.34 & 1.34 & 2.19 & 1.25 & 2.24 & 1.22 & 2.29 & 0.62 \\
\hline Fear & 1.36 & 0.61 & 1.24 & 0.43 & 1.29 & 0.65 & 1.28 & 0.56 & 1.34 & 0.63 & 1.30 & 0.21 \\
\hline Shame & 1.60 & 0.90 & 1.59 & 0.88 & 1.52 & 0.90 & 1.40 & 0.84 & 1.57 & 0.88 & 1.53 & 0.34 \\
\hline Hopel. & 1.48 & 0.80 & 1.52 & 0.86 & 1.72 & 1.07 & 1.76 & 1.08 & 1.67 & 1.07 & 1.63 & 0.40 \\
\hline Bored & 2.47 & 1.16 & 2.69 & 1.13 & 2.66 & 1.37 & 2.64 & 1.44 & 2.57 & 1.42 & 2.60 & 0.69 \\
\hline Surp. & 1.90 & 1.02 & 2.03 & 1.14 & 1.43 & 0.70 & 1.66 & 0.89 & 1.52 & 0.80 & 1.71 & 0.56 \\
\hline Cntmpt. & 1.84 & 1.14 & 1.78 & 1.12 & 1.76 & 1.16 & 1.95 & 1.18 & 1.72 & 1.18 & 1.81 & 0.42 \\
\hline Disgust & 1.16 & 0.37 & 1.26 & 0.55 & 1.21 & 0.55 & 1.22 & 0.56 & 1.34 & 0.69 & 1.24 & 0.17 \\
\hline Confus. & 1.91 & 0.94 & 2.10 & 1.13 & 2.09 & 1.11 & 1.76 & 0.98 & 1.72 & 0.99 & 1.92 & 0.52 \\
\hline Curios. & 3.57 & 1.06 & 3.05 & 1.23 & 2.86 & 1.15 & 2.71 & 1.24 & 2.48 & 1.20 & 2.93 & 0.71 \\
\hline Sad & 1.26 & 0.55 & 1.36 & 0.64 & 1.28 & 0.59 & 1.28 & 0.56 & 1.44 & 0.78 & 1.32 & 0.25 \\
\hline Eureka & 1.50 & 0.78 & 1.74 & 1.09 & 1.66 & 0.98 & 1.67 & 1.05 & 1.57 & 0.98 & 1.63 & 0.34 \\
\hline Neutral & 3.88 & 1.04 & 3.26 & 1.25 & 3.24 & 1.26 & 3.31 & 1.25 & 3.12 & 1.30 & 3.36 & 0.64 \\
\hline
\end{tabular}

Table 2. Summary of Significant Repeated Measure ANOVA Results Using EVs

\begin{tabular}{|c|c|c|c|c|c|c|c|c|c|c|c|c|c|c|}
\hline \multirow[t]{2}{*}{ Emot. } & \multirow[t]{2}{*}{$d f$} & \multirow[t]{2}{*}{$F$} & \multirow[t]{2}{*}{$P$} & \multirow[t]{2}{*}{$\eta_{p}^{2}$} & \multicolumn{10}{|c|}{$\begin{array}{c}\text { Pairwise difference } \\
\quad(p<.05) ?\end{array}$} \\
\hline & & & & & 1,2 & 1,3 & 1,4 & 1,5 & 2,3 & 2,4 & 2,5 & 3,4 & 3,5 & 4,5 \\
\hline Happy & $3.2,177.9$ & 5.77 & $0.01 *$ & 0.09 & & $>$ & $>$ & $>$ & & & & & & \\
\hline Enjoy. & 4,224 & 7.77 & $0.00 *$ & 0.12 & & $>$ & $>$ & $>$ & & & $>$ & & & \\
\hline Hope & $3.3,182.8$ & 15.30 & $0.00 *$ & 0.22 & & $>$ & $>$ & $>$ & & $>$ & $>$ & & & \\
\hline Pride & 4,224 & 3.52 & $0.01 *$ & 0.06 & & & & & & & & & & \\
\hline Anger & 4,224 & 5.76 & $0.00 *$ & 0.09 & & & & $<$ & & & & & & $>$ \\
\hline Frust. & $3.3,184.9$ & 4.57 & $0.00 *$ & 0.08 & & & $<$ & & & $<$ & & & & \\
\hline Surp. & $3.2,179.2$ & 6.54 & $0.00 *$ & 0.11 & & $>$ & & & $>$ & & $>$ & & & \\
\hline Confus. & $.4,224$ & 3.50 & $0.01 *$ & 0.06 & & & & & & & & & & \\
\hline Curios & $3.3,186.6$ & 14.55 & $0.00 *$ & 0.21 & $>$ & $>$ & $>$ & $>$ & & & $>$ & & $>$ & \\
\hline Neutral & 4,224 & 7.32 & $0.00 *$ & 0.12 & $>$ & $>$ & $>$ & $>$ & & & & & & \\
\hline
\end{tabular}

$* \mathrm{p}<0.05$

Note: Greater than signs indicate which emotion's mean for each EV was larger 
that between five and nine of these participants transitioned to a state of surprise or happiness before taking the second EV; the dotted blue line indicates that four or less transitioned to a state of sadness; and the thick, solid blue line indicates that 10 or more also had neutral facial expressions, once again, prior to filling out the EV2.

Table 3. Frequencies and Proportions of Emotions using FaceReader in the 10s before each EV

\begin{tabular}{llllrlrrrrrr}
\hline Emotion & \multicolumn{2}{c}{1} & \multicolumn{2}{c}{2} & \multicolumn{2}{c}{3} & \multicolumn{3}{c}{4} & \multicolumn{2}{c}{5} \\
& \multicolumn{1}{c}{ Freq. } & $\%$ & Freq. & $\%$ & Freq. & $\%$ & Freq. & $\%$ & Freq. & $\%$ \\
\hline Neutral & 45 & 77.6 & 30 & 51.7 & 31 & 53.4 & 33 & 56.9 & 32 & 55.2 \\
Happy & 5 & 8.6 & 11 & 19.0 & 12 & 20.7 & 17 & 29.3 & 11 & 19.0 \\
Surprise & 2 & 3.4 & 7 & 12.1 & 1 & 1.7 & 1 & 1.7 & 3 & 5.2 \\
Fear & - & - & - & - & - & - & - & - & - & - \\
Anger & 2 & 3.4 & - & - & 2 & 3.4 & 2 & 3.4 & 3 & 5.2 \\
Sad & 2 & 3.4 & 4 & 6.9 & 7 & 12.1 & 3 & 5.2 & 3 & 5.2 \\
Disgust & - & - & - & - & - & - & 1 & 1.7 & - & - \\
Unknown & 2 & 3.4 & 6 & 10.3 & 5 & 8.6 & 1 & 1.7 & 6 & 10.3 \\
\hline Sum & 58 & 100 & 58 & 100 & 58 & 100 & 58 & 100 & 58 & 100 \\
\hline
\end{tabular}

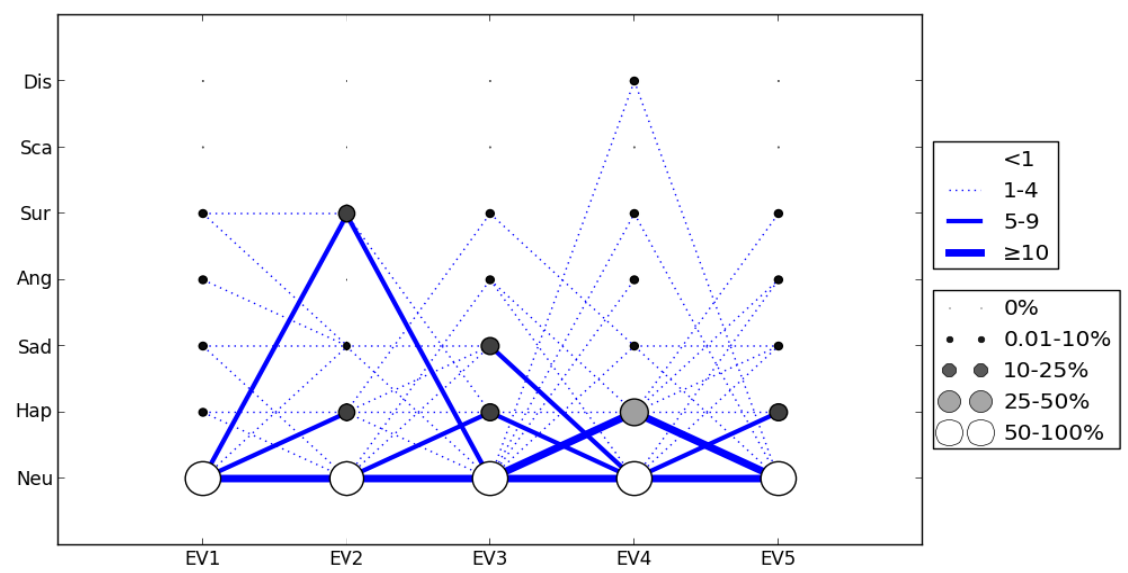

Fig. 1. Transitions between basic emotions using FaceReader data

\subsection{Were There Significant Differences in Learners' Emotional Experiences between MetaTutor's Two PAs Scaffolding Conditions?}

One of the previously described repeated measure ANOVAs revealed a significant main effect of condition on learners' self-reported emotional states: neutral, $F(1,56)$ $=5.87, p<.02, n_{p}^{2}=0.10$. A second repeated measure ANOVA, found a significant interaction effect between $\mathrm{EV}$ and condition for sadness $F(3.01,168.70)=2.73, p<$ $.05, n_{p}^{2}=0.05$. Levene's test of equality of error variances was violated for three of the five EV self-reports for sadness, however, therefore this effect should be interpreted with caution. No other significant effects of condition were found. 


\subsection{Is There Converging Evidence of Learners' Emotional Experiences between Self-report and On-Line Measures?}

Using the method described above to compare self-reported and classified (through FaceReader) emotions, we established an agreement rate ${ }^{1}$ of $75.6 \%$, suggesting that FaceReader can be used reasonably well to assess learner's emotions, even if it cannot provide a fine-grained identification of non-basic (i.e., learner-centered) emotions.

\section{Discussion}

In response to our first research question (which emotions are most prominent in learners' experience with MetaTutor and do they change as the learning session unfolds?) we found that neutral, curiosity and hope had the highest mean levels when averaging all the EVs together. We also noted that of the 19 emotions assessed using the EV, learners' experience of happiness, enjoyment, hope, pride, anger, frustration, surprise, confusion, curiosity, and neutral meaningfully differed across the learning session, while the others remained more stable. In looking at these fluctuations more closely a pattern emerges in which learners' positive, activating emotions and neutral states tended to decline as the session progressed, most notably, between the administration of EV1 and EV3. These patterns draw our attention to a need for an intervention to sustain higher levels of positive emotions (e.g., curiosity, engagement) and neutral states. Another pattern that ran in the opposite direction was the negative, activating emotions anger and frustration, which gradually increased as the session progressed and peaked just before participants filled out the EV4.

In examining the results from FaceReader we observed, similarly, that neutral and a positive activating emotion, happiness, made up the largest proportions of participants' emotional experiences. In particular, most participants embodied a neutral state at each of the EVs, though a substantial proportion of them transitioned to a positive state; the majority of which either transitioned back to a state of neutral or another emotional state before the next EV was administered. It is notable that, similar to the EV self-report analyses in which participants reported low mean levels of negative emotions, few participants facially embodied negative emotions and those who did didn't tend to remain fixed in that state. For example, all of the participants who embodied a sad facial expression before EV3 transitioned to a neutral state before EV4. In summary, these results are favorable, especially considering that MetaTutor is not presently designed using gamification features (e.g., points, story elements) or to provide interventions that specifically aim to improve or sustain learners' (adaptive) emotions. Furthermore, most students were not biology majors ${ }^{2}$ and the content was not designed to be related to a specific course for those who were.

In general, the answer to our second research question, did significant differences in learners' emotional experiences exist between MetaTutor's two PAs

\footnotetext{
${ }^{1}$ Because learners were not asked to provide their dominant emotion among the 19 proposed, it is not possible to provide a kappa value.

${ }^{2} 93 \%$ of students majored in non-biology fields (e.g., psychology, economics, engineering).
} 
scaffolding conditions, is no. Overall, given the low level of negative emotions reported and observed facially, this suggests that at the very least, the more advanced and adaptive feedback that MetaTutor's PAs are providing are not being responded to with negative feelings.

This study also demonstrated that different emotion (behavioral and experiential) measurement methodologies (facial expressions analyses and self-report) can be effectively aligned and produce convergent results. This is particularly notable because of the differences between these two measures. Specifically, the EV assesses the level (e.g., intensity) of a set of potential emotional experiences concurrently, while FaceReader assesses which emotional state learners' are in based on fit with pre-learned facial expressions. Furthermore, these two methods are based on different theories of emotion and use different sub sets of discrete emotions. As a result, despite the strong agreement rate $(75.6 \%)$, there are some differences in terms of the overall patterns, such as the decline in mean levels of positive activating emotions when they are measured separately with the EV vs. the increase in learners' facial expressions of happiness (up to EV 4). This apparent variation in patterns may be the result of subtle differences between the facial embodiment of an emotion and its psychological experience and corresponding self-report. For example, a participant may smile and self-report a 3 on the EV regarding a feeling of pride. In this example, the learner reported experiencing a moderate intensity level of a positive activating emotion (pride) related to FaceReader's classification of happiness as the dominant emotional state, which would be counted as an agreement between the methods.

In conclusion, the high agreement rate we found between methods and convergent results (e.g., that neutral and positively-valenced basic and learner-centered emotional states represented the majority of emotional states experienced with MetaTutor) bolsters the validity of our emotion assessments and provides a strong foundation to make valid and reliable diagnostic examinations of learners' emotions at discrete points during learning with MetaTutor. Conceptually and theoretically, our results provide evidence that the experiential and behavioral components of emotions are tightly coupled. Educationally, improved measurement strategies of emotions will lead to better calibrated interventions that can be designed to support and sustain adaptive emotional states during learning with ITSs.

Acknowledgements. The research presented in this paper has been supported by a doctoral fellowship from the Fonds Québécois de recherche - Société et culture (FQRSC) awarded to the first author and the National Science Foundation (DRL 1008282) awarded to the third author.

\section{References}

1. Chauncey Strain, A., Azevedo, R., D’Mello, S.K.: Using a False Biofeed-back Methodology to Explore Relationships Among Learners' Affect, Metacognition, and Performance. Contemporary Ed. Psych. 38, 22-39 (2013)

2. Pekrun, R., Goetz, T., Frenzel-Anne, C., Petra, B., Perry, R.P.: Measuring Emotions in Students' Learning and Performance: The Achievement Emotions Questionnaire (AEQ). Contemporary Ed. Psych. 36, 34-48 (2011) 
3. D'Mello, S.K., Lehman, B., Graesser, A.: A Motivationally Supportive Affect-Sensitive AutoTutor. In: Calvo, R.A., D’Mello, S.K. (eds.) New Perspectives on Affect and Learning Tech., pp. 113-126. Springer, NY (2011)

4. Robinson, J., McGuiggan, S.W., Lester, J.: Evaluating the Consequences of Affective Feedback in ITSs. In: Cohn, J., Nijholt, A., Pantic, M. (eds.) Proceedings of the 3rd Int. Conference on Affective Computing \& Intelligent Interaction, pp. 37-42. IEEE, Amsterdam (2009)

5. Woolf, B.P., Arroyo, I., Muldner, K., Burleson, W., Cooper, D.G., Dolan, R., Christopherson, R.M.: The Effect of Motivational Learning Companions on Low Achieving Students and Students with Disabilities. In: Aleven, V., Kay, J., Mostow, J. (eds.) ITS 2010, Part I. LNCS, vol. 6094, pp. 327-337. Springer, Heidelberg (2010)

6. Calvo, R.A., D’Mello, S.K. (eds.): New Perspectives on Affect and Learning Technologies. Springer, Amsterdam (2011)

7. Gross, J.J., Barret, L.F.: Emotion Generation and Emotion Regulation: One or Two Depends on Your Point of View. Emotion Rev. 3, 8-16 (2011)

8. VicarVision: FaceReader 5.0 [Computer software]. Noldus Information Technology, Wageningen, The Netherlands (2012)

9. Azevedo, R., Behnagh, R., Duffy, M., Harley, J., Trevors, G.: Metacognition and SRL in Student-Centered Leaning Environments. In: Jonassen, D., Land, S. (eds.) Theoretical Foundations of Student-centered Learning Environments, 2nd edn., pp. 171-197. Routledge, New York (2012)

10. Ekman, P.: An Argument for Basic Emotions. Cognition \& Emotion 6, 169-200 (1992)

11. Terzis, V., Moridis, C.N., Economides, A.A.: Measuring Instant Emotions During a SelfAssessment Test: The Use of FaceReader. In: Proceedings of the 7th Inter. Conf. on Methods and Techniques in Behavioral Research, pp. 18:1-18:4. ACM, New York (2010)

12. D’Mello, S.K., Lehman, B., Person, N.: Monitoring Affective States During Effortful Problem Solving Activities. International Journal of Artificial Intelligence in Education 20, 361-389 (2010)

13. Pekrun, R., Goetz, T., Titz, W., Perry, R.: Academic Achievement Emotions in Students' Self-Regulated Learning and Achievement: A Program of Quantitative and Qualitative Research. Ed. Psychologist 37, 91-206 (2002)

14. Harley, J.M., Bouchet, F., Azevedo, R.: Measuring learners' co-occurring emotional responses during their interaction with a pedagogical agent in MetaTutor. In: Cerri, S.A., Clancey, W.J., Papadourakis, G., Panourgia, K. (eds.) ITS 2012. LNCS, vol. 7315, pp. 40-45. Springer, Heidelberg (2012)

15. Tabachnick, B.G., Fidell, L.S.: Using Multivariate Statistics, 5th edn. Pearson Education/Allyn and Bacon, Boston, MA (2007) 